\title{
Analysis of P300 Related Target Choice in Oddball Paradigm
}

\author{
Yun-Seok Jang, Soo-Ah Ryu, and Kyu-Chil Park, Member, KIMICS
}

\begin{abstract}
P300 component of the event-related brain potential (ERP) reflects fundamental cognitive processes. The $P 300$ components have been employed in a wide variety of theoretical, empirical, and clinical applications. In oddball paradigm for eliciting P300, we need standard stimuli and target stimuli. Firstly, designated stimuli are presented to subjects. Nextly, a chosen target stimulus is presented. In this paper, we elicited the $P 300$ components with experiment that the subjects select a chosen target stimulus. Therefore, we measured the $\mathrm{P300}$ components to be related choice of the target stimuli. The experimental results and the $\mathrm{P300}$ components to be analyzed were presented.
\end{abstract}

Index Terms - P300, Event-related Potential, EEG, Oddball Paradigm, Target, Standard, Amplitude, Latency.

\section{INTRODUCTION}

HUMAN brain activity is related to neurons, glia cells and blood-brain barriers. It is estimated to human brain activity with measurement and analysis of EEG (Electroencephalography). The P300 is one of ERP components getting from EEG. The P300 components have been studied in a wide variety of theoretical, empirical, and clinical applications because the P300 component reflects fundamental cognitive processes. The P300 is a signal that caused by various stimuli to have a specific information. Therefore, the brain activity related to the stimuli can be estimated by analyzing the P300 components.

In the EEG signals, there are many components related to stimuli besides P300s. After the fact that peak of P300 is related to information processing, the research on the P300 has been intensively studied[1].

Since reporting the P300, various experiment methods have been developed to elicit P300. Among the methods, 'oddball paradigm' was a typical method of eliciting P300[2]. P300 is measured using 'oddball paradigm' in which two different stimuli(standard stimulus, target stimulus) are presented in random series. Presenting probability of standard stimulus is high and that of target stimulus is low. The subject is required to distinguish between the two tones by mentally counting or pushing a button responding to the target and not responding to the standard. Studies of P300 using oddball paradigm are

Manuscript received February 7, 2011; revised March 14, 2011; accepted March 28, 2011.

Yun-Seok Jang is with the Dept. of Electrical Engineering, Pukyong National University, Busan, 608-737, Korea (Email: jangys@pknu.ac.kr) consistent with the previous reports. It showed that the target stimuli elicited larger P300 components than the standard stimuli [3]. In topography, P300 amplitude distribution of $\mathrm{Fz}, \mathrm{Cz}, \mathrm{Pz}$ is larger than that of the other electrode[4]. Latency is defined as the time from stimulus onset to the point of maximum positive amplitude. According to many factors, the range of latency(latency window) is from $250 \mathrm{msec}$ to $600 \mathrm{msec}[5]$.

In this study, the P300s were elicited by auditory stimulus using oddball paradigm and analyzed. Generally, standard stimuli and target stimuli are presented to the subjects and we detected and analyzed P300 components when target stimuli were presented. Carrying out the experiment by the method, we have a question about stimulus choice. It is about the relativity between target stimulus choice and P300.

Therefore, we carried out experiments on two ways in this study. Those were to present designated targets and choice targets. We detected P300s from the experiments using the ways as mentioned above and showed the experimental results to analyze the P300s due to target choice in oddball paradigm.

\section{INSTRUMENTATION AND ANALYSIS OF EEG SIGNALS}

\section{A. Experimental Methods}

Seven young adults were served as subjects and all subjects reported being free of neurological or psychiatric disorders. Their ages were from 23 to 25 . The measurement system called PolyG-A by Laxtha was used for recording EEG. The EEG activity was recorded at Fp1, Fp2, F3, Fz, F4, P3, Pz, P4, C3, Cz, C4, T3 and T4 electro sites of the 10-20 system using gold-plated electrodes affixed with electro paste[6]. Linked earlobes were used as the reference with a forehead ground. Auditory stimuli were presented $2000 \mathrm{~Hz}, 1000 \mathrm{~Hz}$ and $500 \mathrm{~Hz}$. Then the stimuli chosen by a subject were presented again. The EEG recording in the first experiment was carried out with typical oddball paradigm. Target stimuli and standard stimuli were presented $2000 \mathrm{~Hz}$ and $1000 \mathrm{~Hz}$ with probability of $0.20,0.80$ respectively in a random series. Because it has been hypothesized that relatively infrequent events elicited large P300 amplitudes[7, 8, 9]. Then it replaced the target stimuli with the standard stimuli in the same way. Lastly the target stimuli chosen by a subject were presented with the same probability as 
above.

In the second experiment, target stimuli were presented $1000 \mathrm{~Hz}$ and $500 \mathrm{~Hz}$ by turns. Then the target stimuli chosen by subject were presented with same probability. In our experiments, 100 stimuli were presented binaurally through headphones at $60 \mathrm{~dB}$ in random series every 2 second. The EEG was sampled by $256 \mathrm{~Hz}$ and the band pass filter was used $12.8 \mathrm{~Hz}$. To remove artifacts from eyes blinking and body movements, the EEG or EOG exceeded $\pm 100 \mu \mathrm{V}$ were rejected automatically. Our experiments were carried out under the condition with eyes closed. The task of the subject was to respond to the target by pushing a button.

\section{B. Experimental Results}

The ERP waveforms elicited by target stimuli are grand averaged. Figures 1 show $\mathrm{P} 300$ peaks by $2000 \mathrm{~Hz}$ target and $1000 \mathrm{~Hz}$ target which were designated. The peak of P300 from $1000 \mathrm{~Hz}$ target is larger than that from $2000 \mathrm{~Hz}$ target. It has been reported that peak latency of P300 component is related to the information processing time and amplitude of that is in proportional to the attentional resource allocation [9]. Due to the report as mentioned above, we can observe that the case of presenting $1000 \mathrm{~Hz}$ target need more attentional resource allocation than that of presenting $2000 \mathrm{~Hz}$ target.
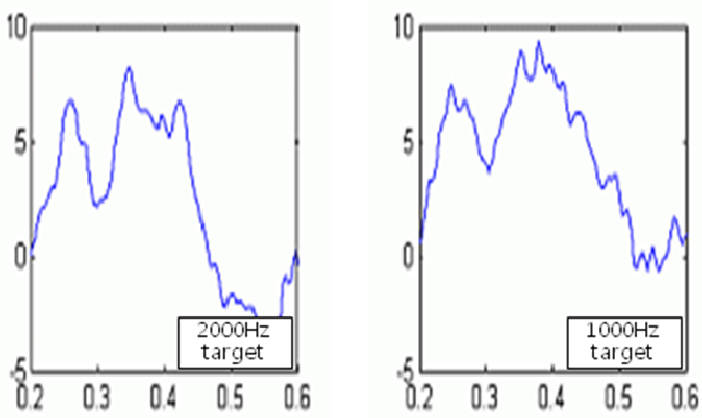

Fig. 1. Grand averaged ERPs from $2000 \mathrm{~Hz}$ target and $1000 \mathrm{~Hz}$ target.

Figure 2 shows the ERP waveforms by $2000 \mathrm{~Hz}$ target and $1000 \mathrm{~Hz}$ target which were chosen by the subjects. Figure 3 shows about the comparison between the P300 amplitudes by designated targets and chosen target by the subject.

Figure 4 shows about the comparison between the P300 latency by designated targets and chosen targets by the subject. By the comparisons, we can observe that the result from the P300 latencies is similar to that from the P300 amplitudes.
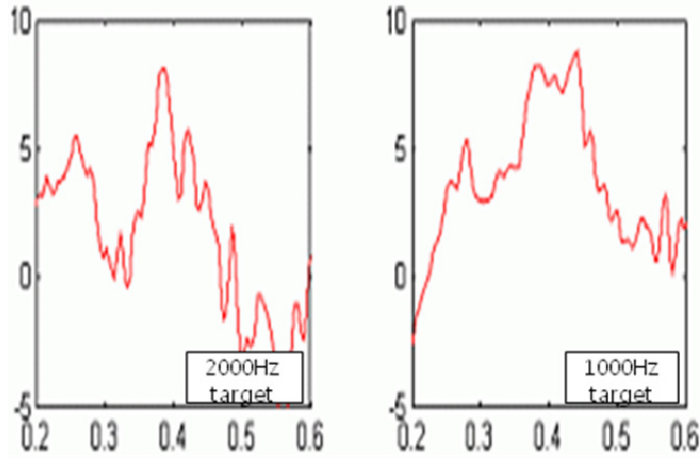

Fig. 2. Grand averaged ERPs from $2000 \mathrm{~Hz}$ target and $1000 \mathrm{~Hz}$ target which were chosen by the subjects.
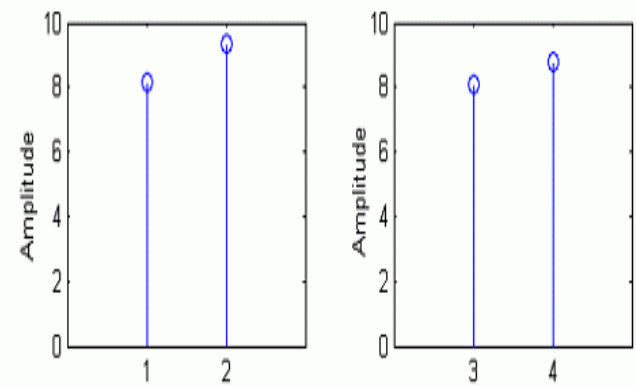

Fig. 3. The comparison between the P300 amplitudes by designated targets $(1,2)$ and chosen targets $(3,4)$.
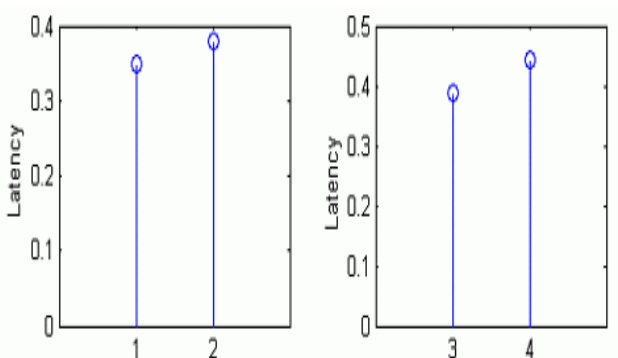

Fig. 4. The comparison between the P300 latency by designated targets $(1,2)$ and chosen targets $(3,4)$.

In the second experiment, figure 5 shows the ERP waveforms by $1000 \mathrm{~Hz}$ target and $500 \mathrm{~Hz}$ target which were designated. 

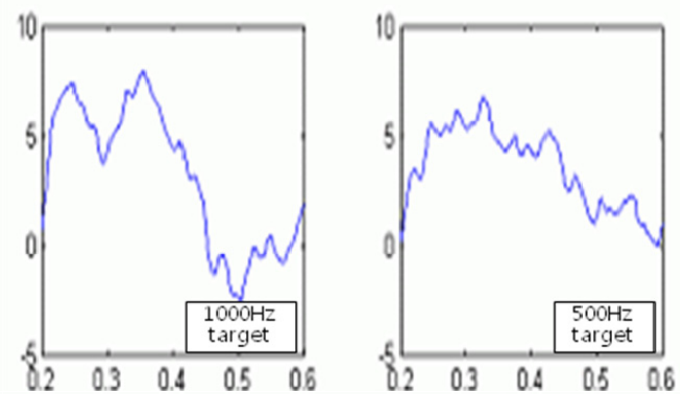

Fig. 5. Grand averaged ERPs from $1000 \mathrm{~Hz}$ target and $500 \mathrm{~Hz}$ target.

Figure 6 shows the ERP waveforms by $1000 \mathrm{~Hz}$ target and $500 \mathrm{~Hz}$ target which were chosen by the subjects.
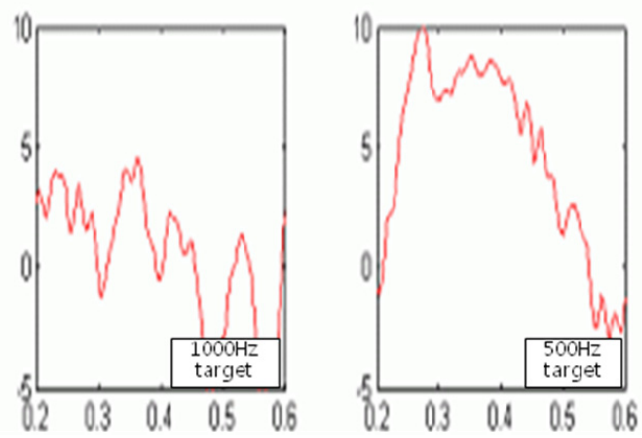

Fig. 6. Grand averaged ERPs from $1000 \mathrm{~Hz}$ target and $500 \mathrm{~Hz}$ target which were chosen by the subjects.
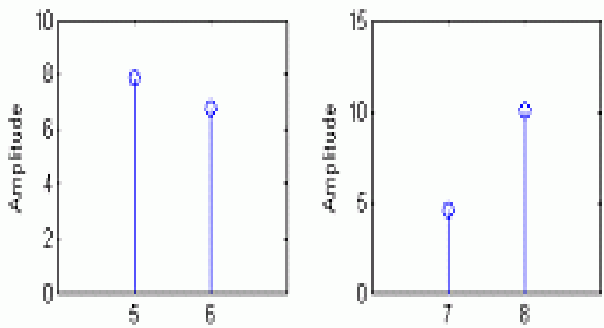

Fig. 7. The comparison between the P300 amplitudes by designated targets $(5,6)$ and chosen targets $(7,8)$.
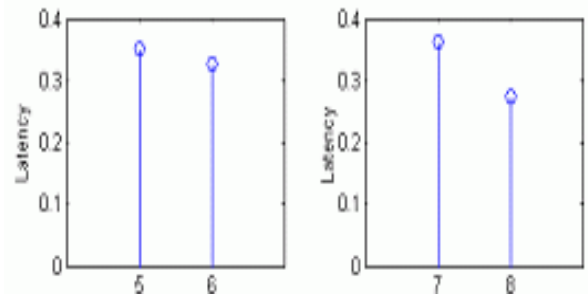

Fig. 8. The comparison between the P300 latency by designated targets $(5,6)$ and chosen targets $(7,8)$.
In the case which $1000 \mathrm{~Hz}$ target is presented (Fig. 1), we can observe the similar P300 waveforms in the results of the first experiment. However, we cannot clearly observe the P300 waveforms in the case of figure 6. In figures comparing the amplitudes, it shows the relations between the amplitudes. Figures comparing the latencies show the relations between the latencies also.

\section{CONCLUSIONS}

We detected and analyzed the P300s elicited using our experimental method proposed in this study. The experimental results show similar features both the target choice case $(1000 \mathrm{~Hz})$ and the non-target choice case $(2000 \mathrm{~Hz})$ in the first experiment. And we can estimate that the attentiveness is more allocated at high-tone stimulus than at low-tone stimulus from the experimental results.

In the second experiment, we could not observe the similar relations in the results of the first experiment. We thought that the target stimulus used in the experiment was not proper as the auditory stimulus.

This findings suggested that the P300s had similar features whether to choose target stimulus or not and the choice of target tone might be needed for the clear P300 peaks.

\section{ACKNOWLEDGMENT}

This work was supported by the Pukyong National University Research Abroad Fund in 2008 (PS-2008-027).

\section{REFERENCES}

[1] Humphery \& Kramer, "Toward a psychophy- siological assessment of dynamic changes in mental workload", Human factors, vol36, pp. 3 26, 1994.

[2] Sutton S., Braren M., \& Zubin J., "Evoked potentials correlates of stimulus uncertainty", Science, vol.150, pp .1187 1188, 1965.

[3] Duncan Johnson, \& Donchin E., "The variation in event-related potentials with subjective probability", Psychophysiology, vol.14, pp.456 467, 1977.

[4] Junichi Katayama, John Polich, "P300 from one- two- threestimilus auditory paradigm", 1996.

[5] Oken B. S., "Endogenous event related potentials", 1989.

[6] Junichi Katayama, John Polich," P300 from one- two- threestimilus auditory paradigm", Int. J. Psychophysiol, vol23, pp. 33 40, 1996.

[7] Brazdil, Rektor, Dufek, Jurak \& Daniel, "Effect of sub threshold target stimuli on event-related potential", Electroencephalography and clinical Neurophysiology, vol.107, pp.64 68, 1998.

[8] Comerchero M. D.,\& Polich J., "P3a and P3b from typical auditory and visual stimuli", Clinical Neurophysiology, vol.110, pp.24 30, 1999.

[9] Brazdil, Rektor, Dufek, Jurak \& Daniel,"Effect of sub threshold target stimuli on event-related potential", Electroencephalography and clinical Neurophysiology, vol.107, pp. 64 68, 1998. 


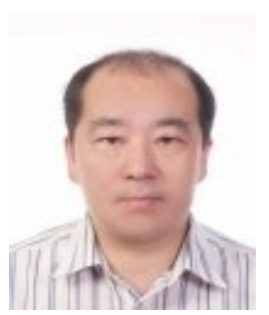

Yun-Seok Jang

Received the B.S. degree in electronics engineering from Busan National University in 1985, and the M.S. degree in electronics engineering from Pukyong National University in 1988 . He received the $\mathrm{Ph}$. D. degree in electricity and communication engineering from Tohoku University of Japan in 1992. Since 1996, he has been a professor in Dept. of Electrical Engineering, Pukyong National University. His research interests include Signal Processing, EEG and Brain Computer Interface.

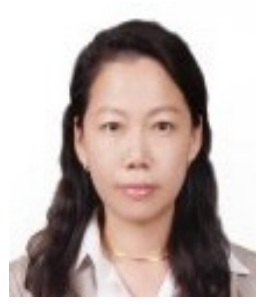

\section{Soo-Ah Ryu}

Received the B.S. degree in Department of Electronics Engineering from Pukyong National University, Busan, Korea in 1991 and the M.S. degree in Department of Telematics Engineering from Pukyong National University in 2007. She is currently Ph.D. degree in Department of Information and Communication Engineering, Pukyong National University, Busan Korea. Her research interests include Signal Processing, EEG and Brain Computer Interface.

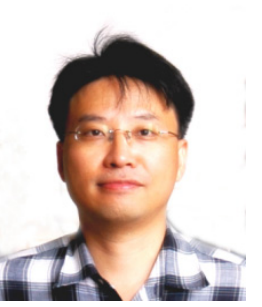

\section{Kyu-Chil Park}

Received the B.S. degree and M.S. degree in Department of Electronic Engineering from Pukyong National University, Busan,, Korea in 1993 and 1995, respectively. He received the Ph.D. degree in Division of Science and Technology for Intelligence, Graduate School of Natural Science and Technology from Okayama University, Okayama, Japan in 2000. Since 2002, he has been a Professor in Department of Information and Communications Engineering, Pukyong National University, Busan, Korea. His research interests include Underwater Acoustic Signal Processing, Adaptive Signal Processing, Numerical Analysis, Optimization and Inverse Problem in Engineering. 